\title{
Endoscopic ultrasound in hepatobiliary hydatid disease
}
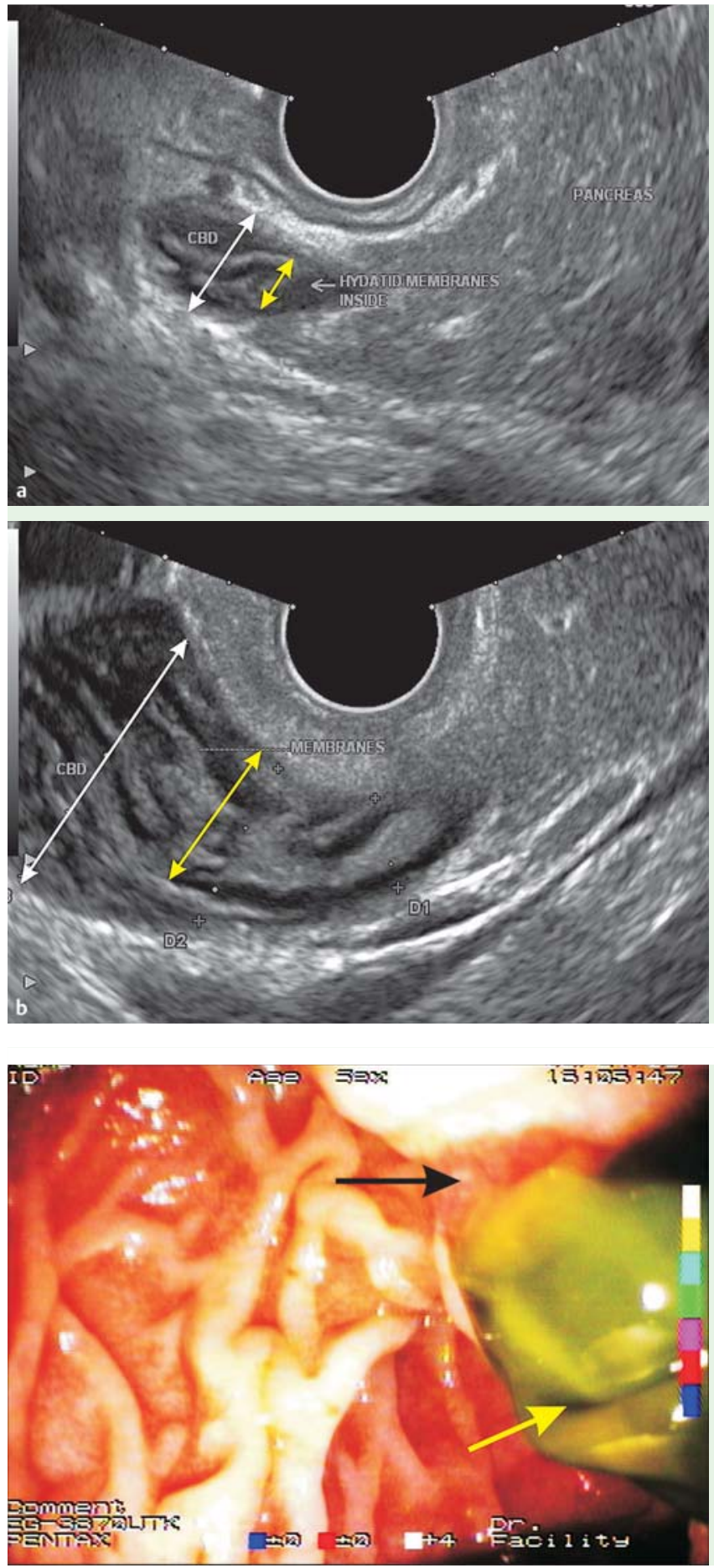

\section{Video 1}

Radial endoscopic ultrasound (EUS) from descending duodenum, demonstrating the dilated common bile duct with hydatid membranes.
Fig. 1 a, b Endoscopic ultrasound (EUS) showing a dilated common bile duct (white arrows) with hyperechoic floating hydatid membranes (yellow arrows).

Fig. 3 Bile-stained hydatid membranes (yellow arrow) extruding out of the ampulla (black arrow) after balloon sweeping of the common bile duct.

\section{Video 2}

Linear endoscopic ultrasound (EUS) from descending duodenum, demonstrating the dilated common bile duct with hydatid membranes.

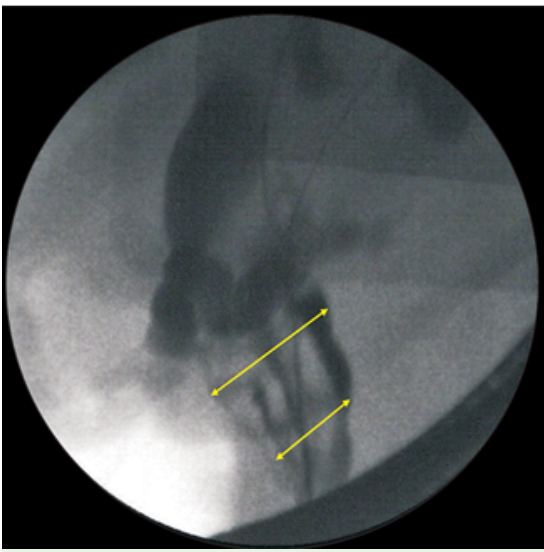

Fig. 2 Cholangiogram demonstrating a dilated common bile duct with multiple linear filling defects suggestive of hydatid membranes.

Rupture into the biliary tract is an unusual complication of hepatic hydatidosis seen in $1-25 \%$ of cases [1]. We present a case of hepatic hydatidosis with biliary rupture identified by endoscopic ultrasound (EUS).

A 45-year-old man presented with a 15day history of fever, right hypochondrial pain, and jaundice. Examination revealed icterus and right hypochondrial tenderness. Investigations revealed polymorphonuclear leukocytosis and a cholestatic pattern of liver function tests. Ultrasound of the abdomen revealed an ill-defined, lobulated, 10.9-cm hyperechoic lesion in the right lobe of the liver, with dilatation of the biliary system up to the lower end of the common bile duct (CBD). The possible diagnoses were choledocholithiasis with cholangiolitic abscess or hepatic hydatidosis. EUS (Pentax 3630 UR and 3870 UTK, Pentax Corp., Tokyo, Japan) revealed a dilated CBD with floating membranes ( Fig. 1 and Videos 1, 2). On endoscopic retrograde cholangiography (ERC), linear filling defects were noted within the dilated CBD ( $\bullet$ Fig. 2 ). Biliary sphincterotomy and balloon sweeping of the CBD resulted in expulsion of the bilestained hydatid membranes ( $\bullet$ Fig. 3). An occlusion cholangiogram showed contrast entering a cavity in the liver ( $\bullet$ Fig. 4). A $7-\mathrm{Fr}$, double pigtail plastic stent was placed close to the cavity in the CBD to facilitate drainage. The patient's condition 


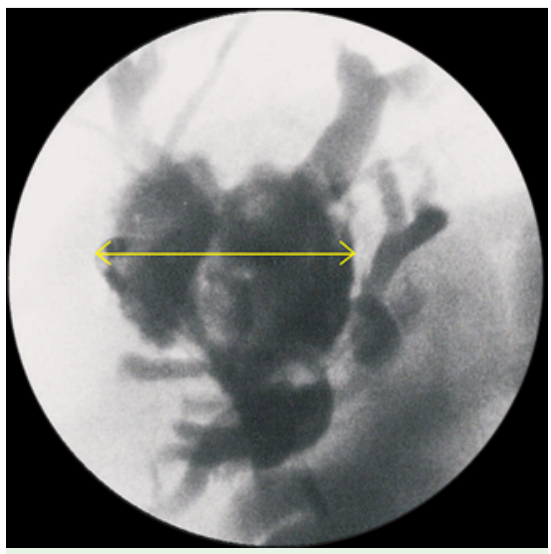

Fig. 4 Cholangiogram showing contrast entering a cystic cavity in the liver.

improved with intravenous antibiotics and albendazole, and a follow-up ultrasound 1 week later revealed a decrease in cyst diameter to $4 \mathrm{~cm}$.

Hydatid disease with biliary rupture may be diagnosed by abdominal ultrasound, computed tomography (CT) or magnetic resonance imaging (MRI) in the presence of clinical features of obstructive jaundice or cholangitis [1,2]. EUS, which can demonstrate the presence of mobile hydatid membranes or cystlike material, may be useful when other imaging modalities are unreliable or unavailable. ERC with sphincterotomy and CBD clearance/stenting has been recommended for managing biliary obstruction and cholangitis [2].

$$
\text { Endoscopy_UCTN_Code_CPL_1AL_2AD }
$$

\section{Sharma ${ }^{1}$, A. Somasundaram ${ }^{1}$,}

A. Pathak', E. G. Simon' ${ }^{2}$, M. M. S. Cloa ${ }^{3}$

1 Department of Gastroenterology, Jaswant Rai Specialty Hospital, Meerut, India

2 Department of Gastrointestinal Sciences, Christian Medical College, Vellore, India

3 Department of Gastroenterology and Digestive Endoscopy, Manila Doctors Hospital, Manila, Philippines

\section{References}

1 Erzurumlu K, Dervisoglu A, Polat $C$ et al. Intrabiliary rupture: an algorithm in the treatment of controversial complication of hepatic hydatidosis. World J Gastroenterol 2005; 11: $2472-2476$

2 Ozaslan E, Bayraktar Y. Endoscopic therapy in the management of hepatobiliary hydatid disease. J Clin Gastroenterol 2002; 35: 160 174

Bibliography

DOI $10.1055 / \mathrm{s}-0029-1243824$

Endoscopy 2010; 42: E56-E57

(c) Georg Thieme Verlag KG Stuttgart · New York . ISSN 0013-726X

Corresponding author

\section{A. Somasundaram}

Department of Gastroenterology

Jaswant Rai Specialty Hospital

Meerut

India

Fax: +91-121-2657154

aravindhsom@gmail.com 